\section{TAB NEWS}

\section{TAB-Bericht zu „Regenerative Energieträger zur Sicherung der Grundlast in der Stromversor- gung“" im Deutschen Bundestag}

Der TAB-Bericht „Regenerative Energieträger zur Sicherung der Grundlast in der Stromversorgung" wurde am 27. Juni 2012 im Ausschuss für Bildung, Forschung und Technikfolgenabschätzung (ABFTA) vorgestellt. Projektleiter Reinhard Grünwald gab einen Überblick über die Projektergebnisse rund um die Kernfrage, wie ein stark ansteigender Anteil meist fluktuierender regenerativer Energiequellen in das Stromsystem integriert und gleichzeitig die Versorgungssicherheit aufrechterhalten werden kann. Gemeinsam mit anderen Triebkräften, u. a. der europäischen Integration der Strommärkte und dem Ausstieg aus der Kernenergie, führt dies dazu, dass die Elektrizitätsversorgung mitten in einem Umbauprozess historischen Ausmaßes steht. Insbesondere muss ihre Flexibilität auf allen Ebenen deutlich erhöht werden, in der Stromerzeugung und bei den Netzen ebenso wie im Bereich der Nachfrage. Für alle Bereiche wurden vom TAB Handlungsoptionen entwickelt und vorgestellt, etwa zu der Frage, wie die öffentliche Hand diesen Prozess unterstützen kann, damit das „Gemeinschaftswerk Energiewende" erfolgreich vorangebracht werden kann.

An die Präsentation schloss sich eine lebhafte Debatte an, in der Redner aus allen Fraktionen hervorhoben, dass der TAB-Bericht eine wertvolle Grundlage für die Parlamentsarbeit in diesem aktuellen Themenfeld darstelle. Zum Abschluss wurde der Bericht vom Ausschuss einstimmig abgenommen und die Veröffentlichung als Bundestagsdrucksache beschlossen. Der Bericht wurde als TAB-Arbeitsbericht Nr. 147 veröffentlicht.

\section{Veranstaltung zu „Frauen und Technik" \\ Von alten Bildern zu neuen Rollen}

Rasante technische Entwicklungen beeinflussen und durchdringen auf vielfältige Art und Weise die Produktions- und Lebenswelten. Bei Entscheidungen in Bezug auf technische Entwicklungen scheinen Frauen jedoch nach wie vor eine untergeordnete Rolle zu spielen. Ist Technik immer noch Männersache? Diese Zuschreibung hält sich auf jeden Fall hartnäckig. Sie hält sich, obwohl der Anteil von Frauen in den Fertigungsbereichen technischer Betriebe schon immer hoch war und Frauen zunehmend auch in der Forschung und Entwicklung als Technikerinnen oder Ingenieurinnen tätig sind. Und sie hält sich weiterhin, obgleich der Gebrauch avancierter Technik inzwischen auch zur „Frauensache“ geworden ist.

Am 23. Mai 2012 fand im Paul-Löbe-Haus eine gemeinsame öffentliche Veranstaltung des Forschungsausschusses, des Deutschen Frauenrats und des TAB - mitverantwortliche Organisatorin war Katrin Gerlinger - statt, die Chancen durch eine stärkere Beteiligung von Frauen bereits bei der Technikentwicklung thematisierte. Nachgegangen wurde dem Mythos einer technischen und einer nichttechnischen Welt, die sich in den Unterschieden zwischen Männern und Frauen traditionellerweise - manifestiert. Gefragt wurde:

- Gibt es tatsächlich einen spezifisch weiblichen Blick auf Technik?

- Verändert sich das Technikdesign durch die größere Teilhabe von Frauen?

- Brauchen wir eine stärkere weibliche Beteiligung und eine bessere Berücksichtigung ihrer Interessen in der Diskussion um technische Entwicklungen und Technologiepolitik?

- Was müsste getan werden, um die höhere Beteiligung der Frauen in technischen Bereichen zu unterstützen?

Die insgesamt sechs Vorträge luden ein, sich ein eigenes Bild zu machen, um im Anschluss gemeinsam zu diskutieren, ob - und wenn ja, welche - Chancen zur Veränderung sich bieten und wo Handlungsräume und -erfordernisse bestehen. 


\section{Delegation des luxemburgi- schen Parlaments im TAB}

Eine Delegation des luxemburgischen Parlaments besuchte am 5. Juli 2012 das TAB. Benoit Reiter, stellvertretender Verwaltungsdirektor der Abgeordnetenkammer (Chambre des Députés du Grand-Duché de Luxembourg), Carlo Linden, Leiter des IT-Referats, sowie Anne Tescher, Sekretärin des Petitionsausschusses, interessierten sich für die Ergebnisse der beiden TAB-Projekte über die Einführung öffentlicher elektronischer Petitionen beim Deutschen Bundestag. Projektleiter Ulrich Riehm konnte in diesem Gespräch auf die grundlegenden Unterschiede zwischen dem Petitionsverfahren des Deutschen Bundestages und der Situation in Luxemburg verweisen, die sich schon allein aus den unterschiedlichen Größenverhältnissen ergeben. Sie sind aber auch darin zu sehen, dass Luxemburg neben dem Petitionsausschuss bei der Volksvertretung auch über einen Bürgerbeauftragten (Ombudsmann) verfügt, dessen Aufgaben in Deutschland auch der Petitionsausschuss des Deutschen Bundestages wahrnimmt.

Das nun im siebten Jahr erfolgreich praktizierte Modell der elektronisch eingereichten und öffentlichen Petitionen des Deutschen Bundestages hat für die Reform des luxemburgischen Petitionsverfahrens eine Vorbildfunktion entwickelt, sodass sich die Fragen der luxemburgischen Delegation in erster Linie auf die Praxiserfahrungen bezogen. Geplant ist, nicht nur die an das Parlament gerichteten Petitionen zu veröffentlichen, sondern auch eine elektronische Mitzeichnung sowie ein Diskussionsforum einzurichten. Diskutiert wurden in diesem Zusammenhang u. a. die Frage der $\mathrm{Zu}$ lassung öffentlicher Petitionen, die Moderation des Diskussionsforums sowie dessen Bedeutung im Petitionsverfahren, Fragen der Authentifizierung sowie des Missbrauchs durch Internetvandalen und die Erfahrungen mit öffentlichen Ausschusssitzungen unter Beteiligung von Petenten zu ausgewählten Petitionen. Das TAB konnte aus seiner umfassenden Forschung zu diesem Thema einen Beitrag zur Meinungsbildung im luxemburgischen Parlament liefern. Die Delegation besuchte neben dem TAB in Berlin auch den Sekretär des Petitionsausschusses des Deutschen Bundestages.

\section{Neue TA-Projekte}

\author{
Inwertsetzung von Biodiversität. Wissenschaft- \\ liche Grundlagen und politische Perspektiven
}

Seit der Rio-Konferenz 1992 der Vereinten Nationen werden Fragen des Schutzes von biologischer Vielfalt auf globaler, europäischer und nationaler Ebene intensiv wissenschaftlich, gesellschaftlich und politisch diskutiert. Verstärkt werden dabei ökonomische Konzepte und Instrumente in den Vordergrund gerückt, um dem Biodiversitätsschutz neue Impulse zu verleihen. Vor dem Hintergrund dieser Entwicklung wurde das TAB mit einer Untersuchung zur „Inwertsetzung von Biodiversität“ beauftragt. Die folgenden drei Themen sollen vertieft bearbeitet werden, mit dem Ziel, Desiderate für die deutsche Forschungslandschaft zu identifizieren sowie die im Hinblick auf politischen Handlungsbedarf kritischen Punkte herauszuarbeiten:

- Analyse des gesellschaftlichen und politischen Diskurses (vor dem Hintergrund von „Rio $+20^{\circ)}$ )

- Grenzen und Reichweite der ökonomischen Bewertung von Biodiversität

- Potenziale und Probleme finanzbasierter Anreizmethoden: Lehren aus dem Klimaschutz?

\section{Medikamente für Afrika. Maßnahmen zur Ver- besserung der gesundheitlichen Situation}

Etwa ein Drittel der Weltbevölkerung hat keinen zuverlässigen Zugang zu wirksamen Medikamenten und ist in besonderem Maße von armutsassoziierten Krankheiten betroffen. Die Gründe dafür sind vielschichtig. Der Fokus des TA-Projekts liegt auf neuen Ansätzen und Konzepten, um die Bedürfnisse der ärmsten Länder stärker im pharmakologischen Innovations- und Produktionsprozess zu berücksichtigen, sowie auf handelspolitischen Rahmenbedingungen und ihren gesundheitsbezogenen Auswirkungen.

Ziel des Projekts ist eine breit angelegte Bestandsaufnahme gegenwärtiger Entwicklungen im Bereich Forschung, Entwicklung und Bereitstellung von Medikamenten für die ärmsten Länder der Erde. Auch wird eine Analyse des Istzustands $\mathrm{zu}$ den relevanten handelspolitischen Vereinbarungen durchgeführt. Auf dieser Basis sollen 
Möglichkeiten für eine zielgerichtete Unterstützung und Ergänzung kommerzieller pharmakologischer Forschungsstrukturen eruiert werden.

\section{Chancen und Kriterien eines Nachhaltig- keitssiegels für Verbraucher}

Der stetig ansteigende Umsatz an ökologisch bzw. nachhaltig erzeugten Waren wie auch die Ergebnisse von Umfragen zur Bereitschaft zum Kauf „nachhaltiger Produkte“ verdeutlichen, dass viele Konsumenten prinzipiell gegenüber nachhaltig erzeugten Produkten und Dienstleistungen aufgeschlossen sind. Zugleich ist die Anzahl an sog. „Marken“, „Siegeln“ und „Zertifikaten" für Waren und Dienstleistungen fast jeglicher Art sehr stark angestiegen, und für Verbraucher wird es immer schwieriger, sich in diesem „Labeldickicht“ zu orientieren. Doch um informierte Konsumentscheidungen treffen $\mathrm{zu}$ können, müssen Verbraucher eindeutig, schnell und zuverlässig erkennen können, was tatsächlich in einem Produkt steckt oder sich hinter einer Dienstleistung verbirgt.

Vor diesem Hintergrund wird in Politik, Verbänden und Wirtschaft diskutiert, ob ein übergreifendes Siegel, das alle Kriterien der Nachhaltigkeit berücksichtigt, Abhilfe schaffen könnte. Umstritten ist, was genau ein solches Siegel abzubilden hätte und welche Kriterien erfüllt sein müssen. Ziel dieses TA-Projekts ist nicht die Erarbeitung eines neuen bzw. eigenen Konzepts für ein Nachhaltigkeitssiegel. Vielmehr sollen eine strukturierte Analyse und Darstellung der Diskussionslage, eine entsprechende Einordnung wichtiger vorliegender Konzepte und Vorschläge und somit letztlich eine fundierte Bewertung der Potenziale und Probleme für ein übergeordnetes Nachhaltigkeitssiegel erfolgen.

\section{$\ll »$}

\section{Neuer Mitarbeiter im TAB}

Maik Poetzsch ist seit Mai 2012 neuer Mitarbeiter des TAB. Er studierte Politikwissenschaft an der Universität Bielefeld (B.A.) und der Freien Universität Berlin (M.A.). In seinem akademischen Werdegang spezialisierte er sich auf die sozialwissenschaftliche Analyse großtechnischer Systeme. Durch seine Tätigkeit als wissenschaftliche Hilfskraft sowie als freier Mitarbeiter für das TAB hat er sich in den letzten Jahren mit den spezifischen Fragestellungen und Arbeitsweisen der Technikfolgenabschätzung vertraut gemacht, u. a. im Rahmen des Projekts „Gefährdung und Verletzbarkeit moderner Gesellschaften - am Beispiel eines großräumigen und langandauernden Ausfalls der Stromversorgung". Aktuell wirkt er an den Projekten „Nachhaltigkeit und Parlamente - Bilanz und Perspektiven Rio+20“ sowie „Chancen und Kriterien eines Nachhaltigkeitssiegels für Verbraucher" mit. Zugleich hat er sein politikwissenschaftliches Promotionsvorhaben an der FU Berlin mit dem Thema „Versorgungssicherheit elektrischer Netze" begonnen.

《»

\section{Weitere TAB-Berichte im Bundestag}

Der TAB-Arbeitsbericht Nr. 141 „Gefährdung und Verletzbarkeit moderner Gesellschaften am Beispiel eines großräumigen und langandauernden Ausfalls der Stromversorgung" wurde am 1. März im Plenum des Deutschen Bundestages diskutiert (Plenarprotokoll 17/162). Die Ausgewogenheit des Berichts wurde hervorgehoben. Er hätte erheblichen Anteil, daran dass dem Thema zivile Sicherheit Deutschlands erstmals ein eigener Tagesordnungspunkt im Plenum gewidmet wurde. In Übereinstimmung mit den Schlussfolgerungen des TAB-Berichts wurde einhellig neben der Stärkung verfügbarer Strukturen und Einsatzkräfte auch eine Stärkung der Sicherheits- und Katastrophenschutzforschung gefordert. Dies sei national wie international geboten. 


\section{Neue Veröffentlichungen}

TAB-Arbeitsbericht Nr. 147 ,,Regenerative Energieträger zur Sicherung der Grundlast in der Stromversorgung “ (April 2012; Verfasser: Reinhard Grünwald, Mario Ragwitz, Frank Sensfuß, Jenny Winkler)

Der Anteil erneuerbarer Energien an der Stromversorgung Deutschlands steigt in den letzten Jahren mit beeindruckender Geschwindigkeit: Er beträgt bereits über 20 Prozent, davon etwa die Hälfte aus fluktuierenden Quellen - v. a. Windkraft und Photovoltaik. Langfristig (bis 2050) wird die Zielsetzung einer nahezu Vollversorgung mit erneuerbaren Energien verfolgt. Damit wird deutlich, dass das System der Stromversorgung in den nächsten Jahrzehnten einem Umbruch historischen Ausmaßes unterliegen wird.

Der TAB-Bericht geht der Frage nach, wie unter diesen Bedingungen die Grundlast in der Stromversorgung weiterhin gesichert werden kann. Diese Frage kann nur in einer Systemperspektive angegangen werden, die alle Ebenen umfasst: von der Erzeugung über den Transport und die Verteilung bis hin zum Verbrauch von Elektrizität. Daher erweitert sich die Fragestellung dahingehend, wie eine gesicherte Versorgung insgesamt organisiert werden kann.

Es zeigt sich, dass das Stromsystem wesentlich flexibler als bisher auf unterschiedliche Einspeise- und Nachfragesituationen reagieren können muss. Optionen zur Steigerung der Flexibilität existieren in vielen Bereichen:

- Erhöhung der Leistungsfähigkeit der Netze

- Flexibilisierung des konventionellen Kraftwerksparks und dessen Betriebsweise

- stärkere Orientierung der Stromproduktion aus erneuerbaren Energien an der Nachfrage

- Lastmanagement, sowie nicht zuletzt

- Errichtung von zusätzlichen Speichern

In allen diesen Handlungsfeldern werden im TAB-Bericht Handlungsoptionen identifiziert, wie die öffentliche Hand bzw. die energiepolitischen Akteure in Exekutive und Legislative durch Gestaltung von Rahmenbedingungen dazu beitragen können, dass der anstehende Umbau der Stromversorgung gelingen kann.
TAB-Arbeitsbericht Nr. 148 ,,Tätigkeitsbericht 2011“ (Juli 2012)

Der Tätigkeitsbericht 2011 des TAB ist erschienen. Er enthält eine Darlegung von Zielen, Inhalten, Zwischenständen und Ergebnissen der im Berichtszeitraum laufenden oder abgeschlossenen Projekte. Darüber hinaus werden Informationen zu den Aufgaben, zur Organisation, zu europäischen Kooperationen und zu den Publikationen des TAB geboten.

Armin Grunwald, Christoph Revermann, Arnold Sauter: Wissen für das Parlament. 20 Jahre Technikfolgenabschätzung am Deutschen Bundestag. Berlin: edition sigma, 2012, 186 S., ISBN 9783836035873, 17,90€

Technikfolgenabschätzung (TA) in Gestalt einer institutionalisierten Beratungseinrichtung für den Deutschen Bundestag blickt mittlerweile auf eine über 20-jährige Tradition zurück. In diesem Zeitraum haben sich die Rahmenbedingungen und Determinanten wissenschaftlicher Politikberatung sichtbar gewandelt. Das Büro für TechnikfolgenAbschätzung beim Deutschen Bundestag (TAB) stand - und steht - vor der Herausforderung, diesen Wandel zu reflektieren und darauf zu reagieren, indem es die komplexer werdenden Mechanismen der Meinungsbildung und Entscheidungsfindung konzeptionell aufgreift und den Veränderungen der demokratischen Entscheidungsprozesse in Gesellschaft und Politik Rechnung trägt. Dieser im August 2012 im Verlag edition sigma erschienene Band spiegelt die Möglichkeiten, aber auch die Schwierigkeiten der TA als Politikberatung durch Aufsätze maßgeblicher Mitgestalter der TA-Landschaft wider. Dabei kommen Autoren aus dem TAB und von außerhalb zu Wort; geboten werden zudem historische Perspektiven und aktuelle Analysen. Die Entstehung des Buches geht auf zwei Anlässe zurück: den 20. Geburtstag des TAB und das Ausscheiden Thomas Petermanns, der seit Gründung des TAB dessen stellvertretender Leiter war und Ende 2011 in den Ruhestand verabschiedet wurde.

\section{$\ll »$}

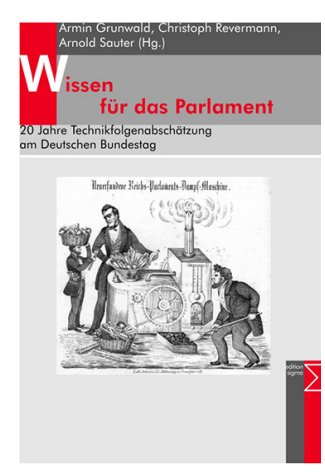

